\title{
Problematização no ensino de gestão em serviços de saúde: experiência de construção de tecnologia de educação
}

\author{
Problematization in health education management teaching: education technology \\ construction experience
}

\section{Problematización en la enseñanza de gestión en servicios de salud: experiencia de construcción de tecnología de educación}

\begin{abstract}
Melissa Barbosa Martins ${ }^{1 *}$, Elielson Paiva Sousa ${ }^{1}$, Viviane Albuquerque Farias ${ }^{1}$, Jessica Soares Barbosa $^{1}$, Jayme Renato Maia Abreu Cordeiro ${ }^{1}$, Glenda Roberta Oliveira Naiff Ferreira ${ }^{1}$.
\end{abstract}

\section{RESUMO}

Objetivo: Descrever experiência prática da construção de uma tecnologia de educação para gestantes utilizando uma metodologia problematizadora. Relato da experiência: Trata-se de um estudo do tipo relato de experiência desenvolvido através da metodologia problematizadora do Arco Maguerez; seguindo as etapas de observação da realidade, identificação de pontos-chaves, teorização, hipótese de solução e aplicação na realidade proposta pelos pontos de assistência da rede cegonha. A observação sucedeu através do levantamento de dados do sistema de informação, diálogos com gestores, usuários e profissionais de saúde e revisão de protocolos. Os pontos-chaves configuraram a fragilidade do acesso à assistência, desconhecimento das gestantes quanto à maternidade conforme classificação de risco; demanda equivocada entre a classificação de risco e complexidade do serviço. A teorização se verificou pelo levantamento de evidências científicas, manuais, normas e portarias para mensurar e explicar o problema. A intervenção foi a elaboração e aplicação de uma cartilha sobre os fluxos da rede cegonha com base na realidade local. Considerações finais: A experiência proporcionou aos discentes reflexões sobre o cotidiano da gestão, atenção e educação na saúde no seu território e contexto nacional ao passo que corrobora a necessidade do docente ao conhecimento, integração e articulação fomentadoras de intervenções em problemas reais.

Palavras-Chave: Acesso aos serviços de saúde, Tecnologia educacional, Enfermagem.

\begin{abstract}
Objective: Describe the practical experience of building an education technology for pregnant women using a problematizing methodology. Experience report: This is an experience report study developed through the problematic methodology of the Maguerez Arch; following the steps of observation of reality, identification of key points, theorizing, hypothesis of solution and application in the reality proposed by the assistance points of the Stork Network. The observation happened through the collection of data from the information system, dialogues with managers, users and health professionals and review of protocols. The key points configured the fragility of access to assistance, the pregnant women's lack of knowledge about maternity according to the risk classification; mistaken demand between risk classification and service complexity. Theorization was verified by the survey of scientific evidence, manuals, rules, and ordinances to measure and explain the problem. The intervention was the development and application of a booklet on the flows of the stork network based on the local reality. Final considerations: The experience provided students with reflections about dayto-day management, care and health education in their territory and the national context while corroborating the teacher's need for knowledge, integration and, an articulation that promote interventions in real problems.
\end{abstract}

Key words: Health services accessibility, Educational technology, Nursing.

1 Universidade Federal do Pará (UFPA), Belém - PA. `E-mail: melissabarbosamartins@gmail.com SUBMETIDO EM: 3/2020 


\section{RESUMEN}

Objetivo: Describir la experiencia de la construcción de una tecnología de educación para gestantes utilizando una metodología problematizadora. Informe de experiencia: Se trata de un estudio de tipo relato de experiencias, desarrollado a través de la metodología problematizadora del Arco Maguerez; siguiendo las etapas de observación de la realidad, identificación de los puntos clave, teorización, hipotesis de solución y aplicación en la realidad propuesta por los puntos de asistencia de la red cigüeña. Resultados: La observación se dio a través de la recopilación de datos del sistema de información, diálogos con los gestores, usuarios y profesionales de la salud y la revisión de protocolos. Los puntos clave configuraron la fragilidad del acceso a la asistencia, desconocimiento de las gestantes con relación a la maternidad conforme a la clasificación de riesgo; demanda y la complejidad del servicio. La intervención fue la elaboración y aplicación de una cartilla sobre los flujos de la red cigüeñas basado en la realidad local. Consideraciones finales: La experiencia proporcionó a los estudiantes reflexiones sobre la cotidianidad de la gestión, atención y educación en salud de su territorio y del contexto nacional mientras corrobora la necesidad del maestro de conocimiento, integración y articulación que fomenta las intervenciones en problemas reales.

Palabras clave: Accesibilidad a los servicios de salud, Tecnología educacional, Enfermaría.

\section{INTRODUÇÃO}

As mudanças nos paradigmas da saúde e educacionais que ocorreram no Brasil nas últimas décadas conduziram os currículos no Brasil a incorporar as mudanças ocorridas na política de formação de recursos humanos do Sistema Único de Saúde (SUS), que requer uma integração mais efetiva das instituições formadoras para atender seus princípios e diretrizes, não apenas como campo de estágio ou de aprendizagem prática, mas para proporcionar uma formação com base em competências que permitam aos trabalhadores participarem ativamente do processo de produção do cuidado e dar respostas as necessidades de saúde da população (VENDRUSCOLO C, et al., 2016).

Nesse contexto e para cumprir o estabelecido nas diretrizes curriculares nacionais (DCN) a utilização das metodologias ativas de ensino aprendizagem (MAEA) rompem com as metodologias tradicionais por possibilitarem uma aproximação entre teoria e prática, formação crítica e reflexiva do estudante/profissional exigida pelas DCN para os cursos da saúde, com formação de um discente autônomo, capaz de construir uma aprendizagem significativa (BRASIL, 2011; BERBEL NAN, 2011; SIMON E, et al., 2014).

A atividade curricular de gestão em serviços de saúde, ministrada no quinto semestre do curso de Enfermagem, busca atender ao perfil de formação estabelecido nas DCN, a ordenação de formação para recursos humanos do SUS e o projeto político pedagógico do Curso de Graduação em Enfermagem de uma Universidade Federal da região Norte do Brasil (BRASIL, 2008).

A abordagem problematizadora contribui para esse perfil de egresso, em que o docente tem papel de mediador do processo ensino-aprendizagem, instrumentalizando o discente a realizar uma observação crítica da realidade e seja capaz de identificar problemas associados a gestão dos serviços que interferem no cuidado e acesso a assistência e, consequentemente com a necessidade de saúde do indivíduo, família e da coletividade.

$\mathrm{Na}$ referida atividade curricular os discentes problematizaram a gestão e atenção na rede cegonha, a primeira rede temática instituída no Brasil, de uma região de saúde da Amazônia brasileira, assim como realizaram uma intervenção no problema priorizado (BRASIL, 2011). Desta forma, este estudo tem como objetivo descrever a experiência prática da construção de uma tecnologia de educação para gestantes utilizando uma metodologia problematizadora.

\section{RELATO DE EXPERIÊNCIA}

Este relato de experiência da metodologia problematizadora aplicada para intervenção na realidade que gerou como produto uma tecnologia educacional. Foi adotada a metodologia da Problematização com o Arco de Charles Maguerez (BERBEL NAN, 2011). Os cenários de prática escolhidos foram um centro de atenção 
secundária de saúde da mulher, um hospital para gestação e parto de alto risco da rede cegonha e dois complexos reguladores, todos estes pertencentes ao SUS.

O estudo foi desenvolvido por acadêmicos do curso de graduação em enfermagem e docentes da Faculdade de Enfermagem da Universidade Federal do Pará (UFPA). Os profissionais de saúde dos cenários e gestantes das unidades foram integrados como coadjuvantes. Os discentes foram orientados sobre a atividade de intervenção na primeira aula no primeiro semestre letivo de 2018, no dia 19 de março de 2018, posteriormente, foram distribuídos em 6 grupos, na qual cada um ficou sob orientação de um docente orientador e de um coorientador que mediaram cada etapa do processo de ensino-aprendizagem.

O desenvolvimento dessa atividade problematizadora está em conformidade com o estabelecido no projeto pedagógico do curso de enfermagem da UFPA, encontra-se descrito no plano de ensino das atividades curriculares gestão em serviços de saúde e organização em serviços de saúde, sendo aprovado pelo colegiado do semestre e coordenação do curso.

Inicialmente, os alunos foram orientados a observar a realidade nos diferentes cenários de atuação do enfermeiro na gestão; seguido da identificação dos pontos-chaves, ou seja, observar e analisar nos cenários os problemas comuns; terceiro, levantar os referenciais teóricos sobre o problema observado; quarto, formular as hipóteses de solução do problema; quinto, a aplicação à realidade da tecnologia de intervenção (BERBEL NAN, 2011). O processo de construção da cartilha seguiu metodologia realizada em estudo prévio (SIMON E, et al., 2014)

Para a leitura da realidade o desenvolvimento da atividade considerou os conhecimentos prévios dos discentes, tanto das atividades curriculares cursadas nos semestres anteriores quanto como usuários do SUS, sendo relevante os conhecimentos sobre consulta pré-natal e identificação de risco das gestantes, construídos nas aulas práticas do terceiro semestre. Elementos da aprendizagem significativa foram utilizados, uma vez que os acadêmicos trazem conhecimentos prévios sobre a rede de serviços que atende a gestação e parto de Belém, na atenção primária, secundária e terciária.

A vivência nos primeiros cenários ocorreu durante o primeiro período de aulas práticas, desta forma, possibilitou que a atividade fosse conduzida ao longo do semestre acadêmico. A aproximação com os gestores ocorreu, em visitas técnicas as centrais de regulação do estado e municípios. Para outros foi agendado reunião, para tratar sobre a atividade e buscar informações para entender o contexto. A base de dados de domínio público, sistema de informação hospitalar do SUS (SIH/SUS) possibilitou a extração, dos tipos de partos realizados por maternidade da região metropolitana I e o local de residência das mulheres, para verificar o tipo de demanda nas maternidades que atendiam parto em gestação de alto risco. Os dados foram extraídos através do aplicativo Tabnet, foram considerados os partos: parto normal; parto normal em gestação de alto risco; parto cesariano em gestação de alto risco; parto cesariano; parto cesariano com laqueadura tubária.

Essas atividades conduziram a identificação dos pontos-chaves do problema. Na teorização usaram-se as bases Scientific eletronic library online (SCIELO) e google acadêmico. Foram selecionados artigos publicados entre 2013 a 2018, considerando o período de implantação da rede cegonha no Brasil. Também foram realizadas leituras de portarias e documentos técnicos, incluindo o plano de ação estadual da rede cegonha. Após a escolha da intervenção, para o detalhamento do processo das etapas foi usada a ferramenta plano de ação ou $5 \mathrm{~W} 2 \mathrm{H}$, que consiste em cinco perguntas: What (O que, qual quê?), Who (Quem?), Where (Onde?), When (Quando?), Why (Por quê?), How (Como?), How Much (Quanto?) (TAJRA, 2006). O processo de elaboração da intervenção será detalhado a seguir.

\section{DISCUSSÃO}

A escolha do problema para intervenção foi composta por etapas. A primeira etapa iniciou com a aproximação da realidade durante as aulas práticas de gestão em serviços de saúde nos diferentes cenários; a segunda etapa foi o levantamento dos problemas, no qual cada discente identificou a situação-problema inicial, ou seja, desconfortos em relação a realidade comum a todos os cenários; na terceira etapa o grupo utilizou uma matriz decisória para priorizar o problema que necessitava de intervenção. Na escolha do 
problema o docente apenas conduziu os discentes a problematizarem a realidade e relacionando-o com as temáticas da atividade curricular, perfil de competência do enfermeiro, cumprindo com o estabelecido para o perfil de formação desejado para profissionais da saúde (BERBEL NAN, 2011; SOUZA ATO, et al., 2015). Desta forma, os conteúdos da atividade curricular de gestão envolvidos nessa etapa foram: regiões de saúde, contextualizando as regiões do Pará; as redes de atenção, considerando a rede cegonha e os pontos de atenção nos três níveis de atenção da Metropolitana I e a atuação do Enfermeiro; sistemas de informação em saúde como subsídios para busca de evidência para contextualização dos cenários; regulação do acesso a assistência, relacionando os pontos de atenção da Metropolitana I, protocolos clínicos da atenção primária à saúde e protocolos de regulação, além de entenderem como a regulação funciona na rede cegonha no contexto do Pará.

Consequentemente, a identificação do problema partiu da percepção dos discentes baseados na observação crítica, levantamento de dados em sistemas de informação em saúde, diálogos com gestores, usuários e profissionais de saúde, e em evidências como protocolos de encaminhamento e acolhimento, para assim, conhecer a magnitude, transcendência, vulnerabilidade e custos e decidir o problema que seria priorizado para a intervenção.

A situação-problema inicial escolhida pelos discentes para intervenção foi o desconhecimento das gestantes quanto ao vínculo a maternidade, conforme classificação de risco, tendo como resultado uma demanda espontânea elevada de partos de baixo risco para a unidade de atenção secundária de pré-natal e parto de alto risco, desta forma evidenciando as fragilidades no acesso a assistência. Essa situação fragiliza a integralidade no processo parturitivo, principalmente, quanto ao vínculo e acolhimento (OLIVEIRA EM E CELENTO DD, 2016).

$\mathrm{Na}$ teorização, a partir dos pontos-chaves os discentes realizaram levantamento de evidências científicas, em base de dados online, além de manuais, normas e portarias para mensurar e explicar o problema, além de estratégias de intervenção que podem ser aplicadas adaptando-se a realidade local. Nessa etapa, a imersão dos discentes nos conteúdos teóricos possibilita que o conhecimento prévio tenha um novo significado levando-os a evidenciarem indissociabilidade na relação entre teoria e prática (BERBEL NAN, 2011).

A próxima etapa foi o estabelecimento das hipóteses de soluções para intervenção no problema, sendo avaliada a viabilidade das soluções. A intervenção escolhida foi a elaboração e aplicação de uma cartilha sobre os fluxos da rede cegonha, e em seguida foi elaborada e pactuado os prazos na ferramenta $5 \mathrm{~W} 2 \mathrm{H}$. Essas etapas diferenciam a problematização de outros recursos pedagógicos mais utilizados em universidades, uma vez que o processo se completa com alguma intervenção, tendo um novo significado para uma ação prática concreta na realidade (BERBEL NAN, 2011). A cartilha, como estratégia de intervenção, é um recurso que pode ser utilizado diretamente pelo público-alvo e, também, pelos profissionais de saúde no ensino (CORDEIRO LI, et al., 2017; THOMÉ LCC, et al., 2017). A elaboração de material educativo que prioriza as dúvidas do usuário, pouco reflete os problemas do vínculo e acesso que são enfrentados apenas durante o período do parto (THOMÉ LCC, et al., 2017).

Para a avaliação e validação, a cartilha foi encaminhada para atores sociais que exercem atividades na rede cegonha, sendo docentes, profissionais enfermeiros, um representante da gestão estadual e gestantes usuárias de uma unidade de atenção primária à saúde. Os profissionais sugeriram alterações na linguagem e organização do fluxo e, entre as gestantes participantes, apenas uma teve dificuldade na interpretação do material. Todavia, levou-se em consideração que fatores externos como o barulho e a grande movimentação na sala de espera da Unidade Básica interferiram na concentração durante a leitura. Esse fator pode ter impactado no processo de aprendizagem e entendimento da usuária em relação ao conteúdo da cartilha, uma vez que o ruído proporciona desconforto que pode ser exteriorizado pela irritação e falta de concentração (SERVILHA EAM e DELLATI MA, 2014).

A avaliação por juízes no material elaborado se constitui uma etapa importante para que as cartilhas educativas possuam conteúdo pertinente e válido. Ademais, na cartilha desenvolvida no estudo, existiam diversas figuras, sendo este um fator importante para o fácil entendimento e leitura agradável. Após a etapa 
de validação, a cartilha sofreu alterações, conforme sugestão dos avaliadores/validadores. Essas alterações são comuns na avaliação de material educativo por juízes (CORDEIRO LI, et al., 2017; REBERTE LM, et al., 2012). A cartilha "Os caminhos da atenção à saúde materna e neonatal em uma região de saúde", em sua versão final, tem dimensões de 14,57x 10,54 cm, contem 20 páginas frente e verso com: capa, contracapa (apresentação da equipe e do conteúdo que compõem a cartilha), sumário, seguido do conteúdo sobre classificação de risco gestacional e parto, sobre os fluxos e pontos de atenção da rede Cegonha.

As etapas de elaboração e validação de cartilha para gestante foram similares ao encontrado em estudos anteriores, porém no presente estudo teve um gestor participando do processo de validação da cartilha (REBERTE LM, et al., 2012). Assim a cartilha elaborada foi um instrumento construído para empoderar a mulher quanto a decisão dos caminhos a tomar no momento do parto, e ao utilizá-la para orientar a gestante durante o pré-natal. Nesse contexto, o enfermeiro ao utilizar uma tecnologia educativa tem sua prática ainda mais valorizada, uma vez que participa de "todos os níveis de assistência, viabilizando o acesso, o acolhimento e a resolubilidade, de forma a assegurar à mulher seu direito de ser protagonista do processo parturitivo" (OLIVEIRA EM e CELENTO DD, 2016).

Ponderamos, do mesmo modo, que este estudo pôde contribuir com a formação de discente e melhor prepará-los para atuar no contexto em que estará inserido durante o seu exercício profissional, com vistas à assistência integral e humanizada, voltada a atender a necessidade de saúde da população. $O$ estudo possibilitou o desdobramento para futuras pesquisas a respeito da elaboração e desenvolvimento sobre adequações do fluxo de acesso à assistência à saúde as gestantes e em outras redes de atenção à saúde.

A utilização da metodologia problematizadora para intervenção em um problema da realidade vivida e repleta de significados para os discentes proporcionou uma reflexão sobre o cotidiano da gestão, atenção e educação em saúde no seu território e no contexto nacional, mobilizando competências cognitivas e atitudinais dos discentes.

A experiência de elaboração e implementação da cartilha demonstrou que o material escrito contribui para alcançar as necessidades do público alvo. Desta forma, a construção do perfil de competência dos egressos/profissionais de Enfermagem requer do docente o conhecimento, integração e articulação com abordagens que capacitem o discente a intervir nos problemas reais que sejam relevantes para a sociedade e território no qual está inserido e considerando os princípios do SUS, não apenas um profissional que seguirá protocolos e executará técnicas.

\section{REFERÊNCIAS}

1. BERBEL NAN. As metodologias ativas e a promoção da autonomia de estudantes. Semina: Ciências Sociais e Humanas. 2011; 32(1) 25-40.

2. BRASIL. ME. Resolução CNE/CES n‥3, de 7 de novembro de 2001. Diretrizes Curriculares Nacionais do Curso de Graduação em Enfermagem. Diário Oficial da União. Seção 1; 2001.

3. BRASIL. MS. Portaria n. 4.279, de 30 de dezembro de 2010 . Estabelece diretrizes para a organização da Rede de Atenção à Saúde no âmbito do Sistema Único de Saúde (SUS).

4. BRASIL. MS. Portaria ํo 1.459, de 24 de junho de 2011. Institui no âmbito do Sistema Único de Saúde - SUS a Rede Cegonha.

5. BRASIL. UFPA. ICS: Faculdade de Enfermagem. Projeto Pedagógico do Curso de Enfermagem. Belém, 2008.

6. CASSIANI SHB, et al. The situation of nursing education in Latin America and the Caribbean towards universal health. Rev. Latino-Am. Enfermagem 2017;

7. CORDEIRO LI, et al. Validation of educational booklet for HIV/Aids prevention in older adults. Rev Bras Enferm. 2017; 70(4):775-82.

8. OLIVEIRA EM e CELENTO DD. A temática da rede cegonha e a inserção do enfermeiro nesse contexto. Revista de Saúde. 2016; 07 (1): 33-38.

9. REBERTE LM, et al. O processo de construção de material educativo para a promoção da saúde da gestante. Rev. Latino-Am. 2012;

10. SERVILHA EAM e DELATTI MA. Percepção de ruído em sala de aula por estudantes universitários e suas consequências sobre a qualidade do aprendizado. Audiol Commun Res. 2014;19 (2):138-44.

11. SIMON E, et al. Metodologias ativas de ensino-aprendizagem e educação popular: encontros e desencontros no contexto da formação dos profissionais de saúde. Interface. 2014 ; 18 (Supl 2):1355-1364.

12. SOUSA ATO, et al. A utilização da teoria da aprendizagem significativa no ensino da Enfermagem. Rev. Bras. Enferm. $2015 ;$ 68(4): 713-722. 
13. TAJRA SF. Gestão estratégica na saúde: reflexões e práticas para uma administração voltada para excelência. 1 edição. látria, 2006.

14. TEIXEIRA E, et al. Panorama dos cursos de Graduação em Enfermagem no Brasil na década das Diretrizes Curriculares Nacionais. Rev. bras. enferm. 2013; 66( spe ): 102-110.

15. THOMÉ LCC, et al. Desafios de discentes e gestantes na assistência pré-natal: processo de construção de cartilha educativa. Interdisciplinary Journal of Health Education. 2017; 2(1): 33-44.

16. VENDRUSCOLO C, et al. Reorientação do ensino no SUS: para além do quadrilátero, o prisma da educação. Revista Reflexão e Ação. 2016; 24(3) 246-260. 